\title{
Selection of Optimum Analytical Lines for the Determination of Several Alloyed Elements in Steel Samples in Glow Discharge Optical Emission Spectrometry with Krypton and Argon
}

\author{
Kazuaki WAGATSUMA \\ Institute for Materials Research, Tohoku University, 2-1-1 Katahira, Sendai 980-8577 Japan. E-mail: wagatuma@imr.tohoku.ac.jp \\ (Received on January 5, 2009; accepted on March 16, 2009)
}

\begin{abstract}
The most sensitive emission lines for determination of cobalt, nickel, and vanadium in steel samples were investigated when krypton was employed as an alternative plasma gas in glow discharge optical emission spectrometry. A thorough survey in the emission spectra indicated that more sensitive ionic lines of nickel as well as cobalt, which were free from spectral interference from iron, could be found when using krypton gas instead of argon gas; therefore, the krypton plasma was recommended in the determination of nickel and cobalt in iron-matrix alloy samples. However, the argon plasma yielded more intense ionic lines of vanadium suitable for the vanadium determination in iron-matrix alloy samples. These phenomena could be explained by resonance energy-transfer collisions with the plasma gases which selectively populate different excited levels of these ions between the krypton and the argon plasmas.
\end{abstract}

KEY WORDS: glow discharge optical emission spectrometry; sensitive analytical line; krypton plasma; nickel; cobalt; vanadium.

\section{Introduction}

Several rare metals, such as $\mathrm{Ni}, \mathrm{Cr}, \mathrm{Co}, \mathrm{V}$, and $\mathrm{Mo}$, are being employed as alloyed elements having the content range from 10 to 0.001 mass $\%$ in steel materials, in which they play an important role in the alloy design determining the mechanical and chemical properties, such as strength, heat resistance, and corrosion resistance. On the other hand, saving of rare metals is recently required in metallurgical industries, because of a variety of their uses but reduction in their resources. Therefore, the steel-making process should be further advanced to obtain the desired properties as well as to reduce the amounts of rare metals, which is associated with analytical methods for monitoring the chemical composition precisely.

Glow discharge optical emission spectrometry (GDOES) is extensively employed for the direct analysis of solid samples, because the excitation source has several benefits for the analytical application: rapid sampling through cathode sputtering, minimal sample pretreatment, and wide concentration range. In addition, GD-OES would provide more accurate analytical results under high signalto-background-ratio conditions compared with other plasma sources, because of the low background continuum. Two books edited by Payling et $a l^{1)}$ and by Marcus and Broekaert $^{2}$ have described the overall contents regarding GD-OES.

The characteristics of the GD plasma are largely varied by several discharge parameters, such as voltage, current, plasma gas composition and the pressure. ${ }^{3-7)}$ It has been reported in the GD plasma that the kind of the plasma gas principally determines the spectrum pattern: different kinds of spectral lines are excited depending on the kind of the plasma gas employed. ${ }^{3)}$ Unlike other plasma sources, the GD plasma source is easily maintained without any instrumental modification even when using various plasma gases such as argon, neon, and helium. Whereas argon is usually used in conventional GD-OES, various plasma gases and their mixtures have been thus investigated as alternative plasma gases. ${ }^{8-13)}$ Their observed spectrum patterns were different from each other, in which the difference is not only in their relative intensities but in the kind of emission lines that can be excited; therefore, one should select optimum analytical lines for each plasma gas employed. We discussed the line selection of copper in GD-OES when using argon and neon as the plasma gas, indicating that different emission lines should be employed for their emission analysis. $^{14)}$

In our pervious report, we reported on a large difference in the relative intensities of nickel ionic lines when the plasma gas was selected between krypton and argon. ${ }^{15)}$ This effect could be explained by selective excitation to particular $3 d^{8} 4 p$ excited levels of nickel ion through charge-transfer collisions ${ }^{16)}$ with krypton or argon ion. Such collisions are most likely to take place when the surplus energy before and after the collision is very small; in other words, an energy resonance is needed, ${ }^{17)}$ meaning that different excited levels can be selectively ionized/excited due to the difference in the first ionization potential between krypton and argon. Therefore, an appropriate combination between a 
particular emission line and a plasma gas could improve the detection sensitivity in GD-OES, in the case where this resonance condition is fulfilled for the corresponding excited energy level. In this paper, we represent optimum analytical lines for determination of cobalt, nickel, and vanadium in steel samples when krypton is employed as the plasma gas instead of argon.

\section{Experimental}

The Grimm-style glow discharge lamp ${ }^{18)}$ and the spectrometer system employed ${ }^{19)}$ have been described in our previous papers. The excitation source was in-house made according to the original model by Grimm. ${ }^{20)}$ Table 1 summarizes the apparatus and the experimental conditions in detail.

Iron-cobalt, iron-nickel, and iron-vanadium binary alloy samples (FXS standard reference materials for X-ray fluorescence analysis, the Iron and Steel Institute of Japan) were employed. Their compositions are 0.52 and 1.06 mass $\% \mathrm{Ni}, 0.50$ and 1.02 mass $\%$ Co, and $0.49,1.00$, and 2.04 mass \% V, respectively. A pure iron plate $(99.8 \%$, purity), a pure nickel plate $(99.9 \%)$, a pure cobalt block $(99.9 \%)$, and a pure vanadium plate $(99.99 \%)$ were also prepared as reference samples. It was polished with waterproof emery papers and then rinsed with ethanol. Before the measurement, pre-discharge was carried out for 5-10 min to remove the surface contaminants. High-purity argon (99.99995\%) or krypton (99.9995\%) was employed as the plasma gas. The pressure of the plasma gas was measured at the vacuum port of the glow lamp with a Prani vacuum gauge, whose readings had been corrected for each gas.

\section{Results and Discussion}

We have already conducted a comparative measurement of Ni II emission lines when krypton and argon are em-

Table 1. Apparatus and the operating conditions.

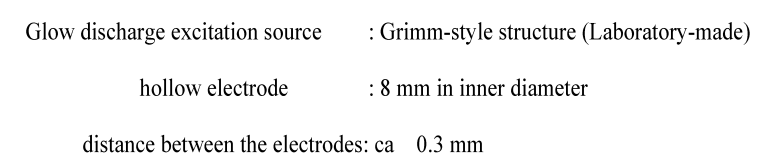

ployed in GD-OES, based on a discussion on the excitation mechanism, and published a wavelength table for the observed Ni II lines. ${ }^{15)}$ The major Ni II lines are assigned to the $3 d^{8} 4 p-3 d^{8} 4 s\left(3 d^{9}\right)$ transition, whose excitation energies range from 6.39 to $9.39 \mathrm{eV}^{21)}$ Among them, particular Ni II lines having an excitation energy of $6.0-6.2 \mathrm{eV}$ can be excited much more dominantly in the krypton plasma as compared with the argon plasma, whereas different Ni II lines can be strongly excited in the argon plasma although they can be observed very little in the krypton plasma. The latter $\mathrm{Ni}$ II lines have an excitation energy of $8.1-8.3 \mathrm{eV}$. These results are because different emission lines are dominantly excited for each plasma gas and their excitation processes could be selectively caused depending on their excitation energies. As a result, calibration curves for the nickel determination are very different between the krypton and the argon plasmas. Figure 1 shows a typical example for the calibration curve, in which Ni II $229.968 \mathrm{~nm}(8.25 \mathrm{eV})$ and $\mathrm{Ni}$ II $230.299 \mathrm{~nm}(6.54 \mathrm{eV})$ are employed as the analytical line. In the Ni II 230.299-nm line, the krypton plasma is much superior to the argon plasma because of the better detection sensitivity. However, in the Ni II 229.968-nm line, the argon plasma gives much better analytical performance than the krypton plasma. It is thus found that the excitation energy: $6.5 \mathrm{eV}$ for the krypton plasma and $8.3 \mathrm{eV}$ for the argon plasma, have a significant meaning for selection of the analytical line in GD-OES. On the other hand, the emission intensities of nickel atomic lines are not so different between the krypton plasma and the argon plasma. A large
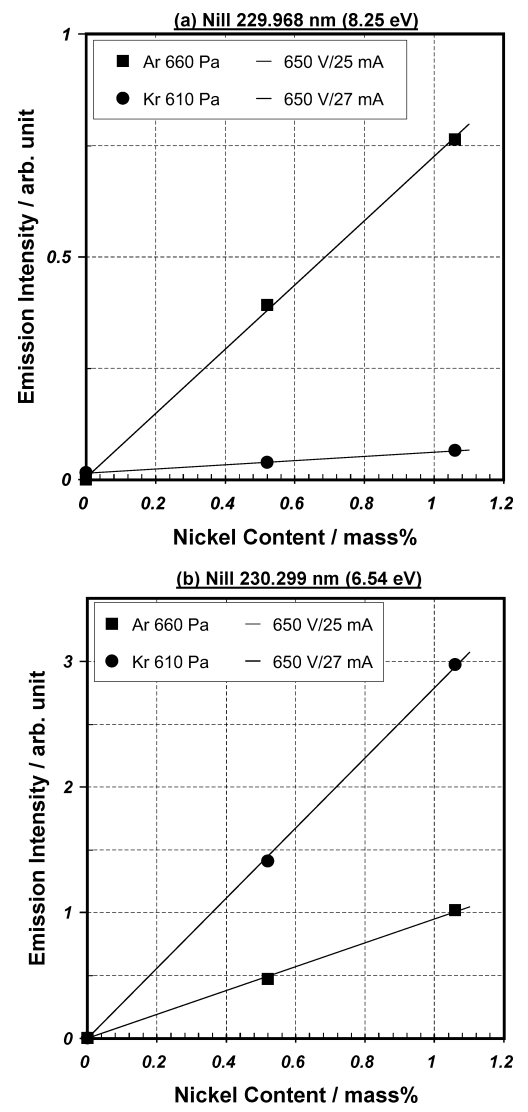

Fig. 1. Calibration curve for the Ni II $229.968 \mathrm{~nm}$ (a) and the Ni II $230.299 \mathrm{~nm}$ in a krypton plasma (circle) and an argon plasma (square). Discharge conditions: $\mathrm{Kr}, 610 \mathrm{~Pa}$ / $650 \mathrm{~V} / 27 \mathrm{~mA} ; \mathrm{Ar}, 660 \mathrm{~Pa} / 650 \mathrm{~V} / 25 \mathrm{~mA}$. 


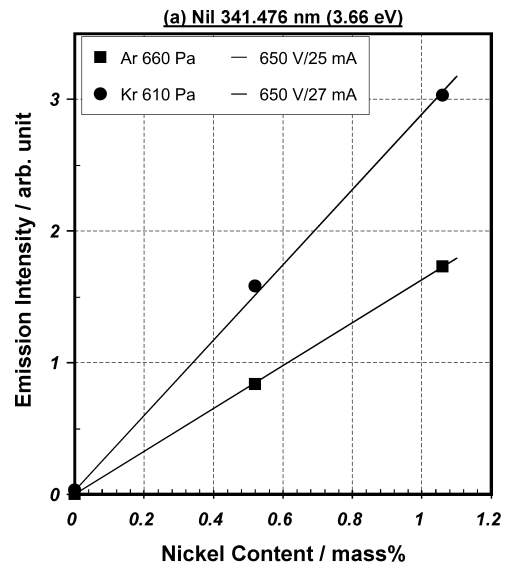

(b) Nil $352.453 \mathrm{~nm}(3.54 \mathrm{eV})$

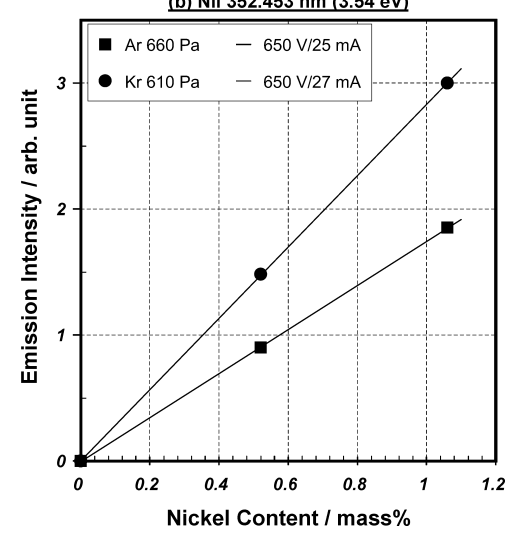

Fig. 2. Calibration curve for the Ni I $341.476 \mathrm{~nm}$ (a) and the Ni I $352.453 \mathrm{~nm}$ in a krypton plasma (circle) and an argon plasma (square). Discharge conditions: $\mathrm{Kr}, 610 \mathrm{~Pa} / 650 \mathrm{~V} /$ $27 \mathrm{~mA}$; Ar, $660 \mathrm{~Pa} / 650 \mathrm{~V} / 25 \mathrm{~mA}$.

number of Ni I lines, which are assigned to the $3 d^{9} 4 p-3 d^{9} 4 s$ transition, appear in the wavelength range of $340-370 \mathrm{~nm}$, and their relative intensities roughly follow the transition probability of each transition. Figure 2 shows the calibration curves for two sensitive Ni I lines, Ni I $341.476 \mathrm{~nm}$ $(3.66 \mathrm{eV})$ and Ni I $352.453 \mathrm{~nm}(3.54 \mathrm{eV})$. It is found in Figs. 1 and 2 that, when the plasma gas is changed from argon to krypton, the sensitivity difference in the Ni I lines is much smaller than that of the Ni II lines.

Cobalt ion has a similar electron configuration to nickel ion but the ionization potential is slightly higher than nickel. ${ }^{21)}$ I have revealed a similarity in the excitation mechanism between nickel and cobalt in detail. ${ }^{22)}$ Table 2 summarizes Co II emission lines observed in the wavelength range of 230-262 nm, when a krypton or an argon discharge is conducted at a gas pressure of $530 \mathrm{~Pa}$ for a pure cobalt sample. They are assigned to the $3 d^{7} 4 p-3 d^{7} 4 s$ $\left(3 d^{8}\right)$ transition whose excitation energies range from 5.60 to $9.23 \mathrm{eV}$. One can find that there is a large difference in the emission intensity between the krypton and the argon plasmas and that the behavior is closely dependent on the excitation energy. For instance, the emission intensity of the Co II $258.033 \mathrm{~nm}(6.02 \mathrm{eV})$ was 220000 in the krypton plasma and 23000 in the argon plasma, whereas the intensity of the Co II $231.707 \mathrm{~nm}(8.08 \mathrm{eV})$ was 300 in the krypton plasma and 22000 in the argon plasma. Figure 3 shows the intensity variations of these Co II lines as a function of the discharge voltage when the pure cobalt sample is em-
Table 2. Observed cobalt ionic lines and their assignment together with the relative intensity in the $\mathrm{Ar}$ and the $\mathrm{Kr}$ plasmas.

\begin{tabular}{|c|c|c|c|c|c|c|}
\hline \multirow{2}{*}{$\begin{array}{c}\text { Wavelength } \\
(\mathbf{n m})\end{array}$} & \multicolumn{4}{|c|}{ Assignment } & \multicolumn{2}{|c|}{ Relative Intensity ${ }^{*}$} \\
\hline & \multicolumn{2}{|c|}{ Upper level (eV) } & \multicolumn{2}{|c|}{ Lower Level (eV) } & \multirow{2}{*}{$\begin{array}{c}\mathbf{K r} \\
10000\end{array}$} & \multirow{2}{*}{$\frac{\text { Ar }}{13000}$} \\
\hline Co I 387.311 & (atomic lin & for the stanc & Ird of normalizat & & & \\
\hline Co II 230.786 & $3 d^{7} 4 p^{5} \mathbf{G}_{5}$ & 5.8700 & $3 d^{7} 4 s^{5} \mathbf{F}_{4}$ & 0.4995 & 32000 & 8000 \\
\hline Co II 231.160 & $3 d^{7} 4 p^{5} \mathbf{G}_{4}$ & 5.9272 & $3 d^{7} 4 s^{5} \mathbf{F}_{3}$ & 0.5655 & 57000 & 6000 \\
\hline Co II 231.361 & $3 d^{7} 4 p^{5} \mathbf{D}_{3}$ & 7.5927 & $3 d^{7} 4 s^{5} \mathbf{P}_{2}$ & 2.2356 & 200 & 5000 \\
\hline Co II 231.406 & $3 d^{7} 4 p^{5} \mathbf{G}_{3}$ & 5.9698 & $3 d^{7} 4 s^{5} \mathbf{F}_{2}$ & 0.6137 & 63000 & 6000 \\
\hline Co II 231.707 & $3 d^{7} 4 p^{3} \mathbf{G}_{4}$ & 8.0779 & $3 d^{7} 4 s^{3} \mathbf{G}_{4}$ & 2.7288 & 300 & 22000 \\
\hline Co II 235.822 & $3 d^{7} 4 p^{1} \mathbf{G}_{4}$ & 7.9846 & $3 d^{7} 4 s^{3} \mathbf{G}_{4}$ & 2.7283 & 300 & 12000 \\
\hline Co II 236.051 & $3 d^{7} 4 p^{3} \mathbf{F}_{3}$ & 7.9795 & $3 d^{7} 4 s^{3} \mathbf{G}_{4}$ & 2.7288 & 400 & 28000 \\
\hline Co II 236.079 & $3 d^{7} 4 p^{3} \mathbf{F}_{3}$ & 8.7355 & $3 d^{7} 4 s^{3} \mathbf{D}_{2}$ & 3.4854 & 100 & 4000 \\
\hline Co II 236.379 & $3 d^{7} 4 p^{5} \mathbf{D}_{4}$ & 5.7429 & $3 d^{7} 4 s^{5} \mathbf{F}_{4}$ & 0.4995 & 6000 & 4000 \\
\hline Co II 237.184 & $3 d^{7} 4 p^{1} \mathbf{I}_{6}$ & 8.6313 & $3 d^{7} 4 s^{3} \mathbf{H}_{5}$ & 3.4056 & 200 & 2000 \\
\hline Co II 237.862 & $3 d^{7} 4 p^{5} \mathbf{F}_{4}$ & 5.6261 & $3 d^{7} 4 s^{5} \mathbf{F}_{5}$ & 0.5154 & 6000 & 4000 \\
\hline Co II 238.175 & $3 d^{7} 4 p^{3} \mathbf{H}_{6}$ & 7.8849 & $3 d^{7} 4 s^{3} \mathbf{G}_{5}$ & 2.6810 & 500 & 51000 \\
\hline Co II 238.671 & $3 d^{7} 4 p^{3} \mathbf{F}_{4}$ & 7.8741 & $3 d^{7} 4 s^{3} \mathbf{G}_{5}$ & 2.6810 & 300 & 21000 \\
\hline Co II 238.891 & $3 d^{7} 4 p^{5} \mathbf{F}_{5}$ & 5.6037 & $3 d^{7} 4 s^{5} \mathbf{F}_{3}$ & 0.4154 & 10000 & 6000 \\
\hline Co II 239.257 & $3 d^{7} 4 p^{3} \mathbf{H}_{4}$ & 7.9091 & $3 d^{7} 4 s^{3} \mathbf{G}_{4}$ & 2.7288 & 300 & 5000 \\
\hline Co II 239.738 & $3 d^{7} 4 p^{3} \mathbf{D}_{3}$ & 6.3866 & $3 d^{7} 4 s^{3} \mathbf{F}_{4}$ & 1.2166 & 7000 & 24000 \\
\hline Co II 240.380 & $3 d^{7} 4 p^{1}$ & 8.7850 & $3 d^{7} 4 s^{3} \mathbf{D}_{1}$ & 3.6288 & 200 & 1000 \\
\hline Co II 240.452 & $3 d^{7} 4 p^{3} \mathbf{D}_{3}$ & 8.5622 & $3 d^{7} 4 s^{3} \mathbf{D}_{3}$ & 3.4076 & 100 & 3000 \\
\hline Co II 240.767 & $3 d^{7} 4 p^{3} \mathbf{D}_{2}$ & 6.4755 & $3 d^{7} 4 s^{3} \mathbf{F}_{3}$ & 1.3277 & 6000 & 21000 \\
\hline Co II 241.408 & $3 d^{7} 4 p^{5} \mathbf{F}_{3}$ & 5.6997 & $3 d^{7} 4 s^{5} \mathbf{F}_{3}$ & 0.5654 & 2000 & 4000 \\
\hline Co II 241.531 & $3 d^{7} 4 p^{3} \mathbf{P}_{1}$ & 8.1403 & $3 d^{7} 4 s^{3} \mathbf{P}_{1}$ & 3.0087 & 4000 & 6000 \\
\hline Co II 241.600 & $3 d^{7} 4 p^{3} \mathbf{H}_{4}$ & 7.9091 & $3 d^{7} 4 s^{3} \mathbf{G}_{3}$ & 2.7790 & 300 & 3000 \\
\hline Co II 241.621 & $3 d^{7} 4 p^{3} \mathbf{G}_{3}$ & 8.5353 & $3 d^{7} 4 s^{3} \mathbf{H}_{5}$ & 3.4056 & 200 & 6000 \\
\hline Co II 241.690 & $3 d^{7} 4 p^{3} \mathbf{D}_{1}$ & 6.5319 & $3 d^{7} 4 s^{3} \mathbf{F}_{2}$ & 1.4037 & 3000 & 20000 \\
\hline Co II 242.073 & $3 d^{7} 4 p^{3} \mathbf{H}_{5}$ & 7.8488 & $3 d^{7} 4 s^{3} \mathbf{G}_{4}$ & 2.7288 & 300 & 32000 \\
\hline Co II 242.383 & $3 d^{7} 4 p^{3} \mathbf{G}_{3}$ & 8.1 & $3 d^{7} 4 s^{3} \mathbf{D}_{2}$ & 3.4854 & 100 & 4000 \\
\hline Co II 243.250 & $3 d^{7} 4 p^{3} \mathbf{G}_{5}$ & 8.4559 & $3 d^{7} 4 s^{3} \mathbf{H}_{6}$ & 3.3606 & 100 & 10000 \\
\hline Co II 244.378 & $3 d^{7} 4 p^{3} \mathbf{D}_{2}$ & 6.4755 & $3 d^{7} 4 s^{3} \mathbf{F}_{2}$ & 1.4037 & 2000 & 7000 \\
\hline Co II 244.602 & $3 d^{7} 4 p^{1} \mathbf{F}_{3}$ & 8.1850 & $3 d^{7} 4 s^{1} \mathbf{G}_{4}$ & 3.1178 & 500 & 17000 \\
\hline Co II 249.883 & $3 d^{7} 4 p^{3} \mathbf{G}_{4}$ & 8.0779 & $3 d^{7} 4 s^{1} \mathbf{G}_{4}$ & 3.1178 & 200 & 11000 \\
\hline Co II 250.646 & $3 d^{7} 4 p^{3} \mathbf{F}_{4}$ & 6.1616 & $3 d^{7} 4 s^{3} \mathbf{F}_{4}$ & 1.2166 & 83000 & 8000 \\
\hline Co II 251.117 & $3 d^{7} 4 p^{1} \mathbf{H}_{5}$ & 8.0535 & $3 d^{7} 4 s^{1} \mathbf{G}_{4}$ & 3.1178 & 4000 & 33000 \\
\hline Co II 251.982 & $3 d^{7} 4 p^{3} \mathbf{F}_{3}$ & 6.2464 & $3 d^{7} 4 s^{3} \mathbf{F}_{3}$ & 1.3277 & 55000 & 5000 \\
\hline Co II 252.296 & $3 d^{7} 4 p \quad 2$ & 8.3327 & $3 d^{7} 4 s^{1} \mathbf{P}_{1}$ & 3.4201 & 100 & 5000 \\
\hline Co II 252.464 & $3 d^{7} 4 p^{3} \mathbf{D}_{1}$ & 7.9181 & $3 d^{7} 4 s^{3} \mathbf{P}_{1}$ & 3.0087 & 300 & 6000 \\
\hline Co II 252.497 & $3 d^{7} 4 p^{3} \mathbf{F}_{2}$ & 6.3124 & $3 d^{7} 4 s^{3} \mathbf{F}_{2}$ & 1.4037 & 4000 & 11000 \\
\hline Co II 252.862 & $3 d^{7} 4 p^{3} \mathbf{G}_{4}$ & 6.1183 & $3 d^{7} 4 s^{3} \mathbf{F}_{4}$ & 1.2166 & 90000 & 9000 \\
\hline Co II 253.009 & $3 d^{7} 4 p^{3} \mathbf{D}_{3}$ & 7.8836 & $3 d^{7} 4 s^{3} \mathbf{P}_{2}$ & 2.9848 & 2000 & 24000 \\
\hline Co II 253.382 & $3 d^{7} 4 p^{3} \mathbf{G}_{5}$ & 8.0094 & $3 d^{7} 4 s^{1} \mathbf{G}_{4}$ & 3.1178 & 300 & 14000 \\
\hline Co II 254.063 & $3 d^{7} 4 p^{3} \mathbf{D}_{2}$ & 7.8872 & $3 d^{7} 4 s^{3} \mathbf{P}_{1}$ & 3.0087 & 400 & 1500 \\
\hline Co II 255.679 & $3 d^{7} 4 p^{5} \mathbf{P}_{2}$ & 7.8564 & $3 d^{7} 4 s^{3} \mathbf{P}_{1}$ & 3.0087 & 300 & 15000 \\
\hline Co II 255.733 & $3 d^{7} 4 p^{3} \mathbf{D}_{1}$ & 6.5319 & $3 d^{7} 4 s^{3} \mathbf{P}_{0}$ & 1.6853 & 400 & 2000 \\
\hline Co II 255.940 & $3 d^{7} 4 p^{3} \mathbf{F}_{3}$ & 6.2464 & $3 d^{7} 4 s^{3} \mathbf{F}_{2}$ & 1.4087 & 47000 & 4000 \\
\hline Co II 256.004 & $3 d^{7} 4 p^{1} \mathbf{I}_{6}$ & 8.6313 & $3 d^{7} 4 s^{1} \mathbf{H}_{5}$ & 3.7898 & 200 & 8000 \\
\hline Co II 256.406 & $3 d^{7} 4 p^{3} \mathbf{F}_{4}$ & 6.1616 & $3 d^{7} 4 s^{3} \mathbf{F}_{3}$ & 1.3277 & 90000 & 9000 \\
\hline Co II 257.486 & $3 d^{7} 4 p^{3} \mathbf{D}_{2}$ & 6.4755 & $3 d^{7} 4 s^{3} \mathbf{P}_{1}$ & 1.6619 & 1000 & 4000 \\
\hline Co II 258.033 & $3 d^{7} 4 p^{3} \mathbf{G}_{5}$ & 6.0201 & $3 d^{7} 4 s^{3} \mathbf{F}_{4}$ & 1.2166 & 220000 & 23000 \\
\hline Co II 258.224 & $3 d^{7} 4 p^{3} \mathbf{G}_{3}$ & 6.2036 & $3 d^{7} 4 s^{3} \mathbf{F}_{2}$ & 1.4037 & 100000 & 10000 \\
\hline Co II 258.318 & $3 d^{7} 4 p^{3} \mathbf{D}_{3}$ & 7.8836 & $3 d^{7} 4 s^{3} \mathbf{P}_{2}$ & 3.0855 & 300 & 12000 \\
\hline Co II 258.685 & $3 d^{7} 4 p^{3} \mathbf{H}_{4}$ & 7.9091 & $3 d^{7} 4 s^{1} \mathbf{G}_{4}$ & 3.1178 & 60 & 2000 \\
\hline Co II 258.723 & $3 d^{7} 4 p^{3} \mathbf{G}_{4}$ & 6.1183 & $3 d^{7} 4 s^{3} \mathbf{F}_{3}$ & 1.3277 & 90000 & 10000 \\
\hline Co II 260.569 & $3 d^{7} 4 p^{3} \mathbf{S}_{1}$ & 7.7415 & $3 d^{7} 4 s^{3} \mathbf{P}_{2}$ & 2.9848 & 300 & 11000 \\
\hline Co II 260.590 & $3 d^{7} 4 p^{3} \mathbf{F}_{4}$ & 7.8741 & $3 d^{7} 4 s^{1} \mathbf{G}_{4}$ & 3.1178 & 200 & 4000 \\
\hline Co II 261.349 & $3 d^{7} 4 p^{3} \mathbf{D}_{3}$ & 6.3866 & $3 d^{7} 4 s^{3} \mathbf{P}_{2}$ & 1.6441 & 1000 & 4000 \\
\hline Co II 261.981 & $3 d^{7} 4 p^{3} \mathbf{H}_{5}$ & 7.8488 & $3 d^{7} 4 s^{1} \mathbf{G}_{4}$ & 3.1178 & 100 & 6000 \\
\hline
\end{tabular}

*) Discharge conditions: Kr $530 \mathrm{~Pa} / 700 \mathrm{~V} / 30 \mathrm{~mA}$; Ar $530 \mathrm{~Pa} / 700 \mathrm{~V} / 29 \mathrm{~mA}$. 

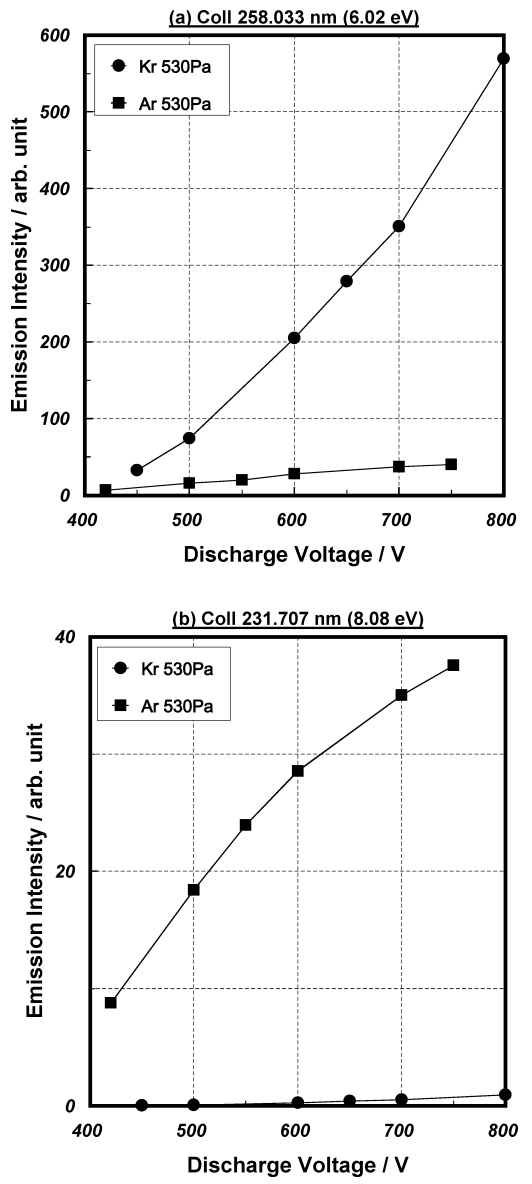

Fig. 3. Variations in the emission intensity of the Co II 258.033 $\mathrm{nm}$ (a) and the Co II $231.707 \mathrm{~nm}$ in a krypton plasma (circle) and an argon plasma (square). Sample: pure cobalt; gas pressure: $\mathrm{Kr} 530 \mathrm{~Pa}$ and $\mathrm{Ar} 530 \mathrm{~Pa}$.

ployed, indicating that the intensity of Co II $258.033 \mathrm{~nm}$ is always larger in the krypton plasma regardless of the discharge voltages, whereas it is hard to observe the Co II 231.707-nm line in the krypton plasma even at higher discharge voltages. Figure 4 shows calibration curves for several Co II lines having different excitation energies which do not suffer from any spectral interference from iron and gas emission lines. A large difference in the detection sensitivity of the Co II 258.033-nm line is observed: the krypton plasma gives much better sensitivity than the argon plasma. On the other hand, the Co II 242.073-nm line can be a little excited from the krypton plasma, resulting in superiority of the argon plasma for this excitation. As similar to the Ni II emission lines, the difference in the emission intensities of these Co II lines can be explained by the selective excitation through resonance energy transfer from plasma gas ions. ${ }^{22)}$ It is found in the case of Co II lines that the excitation energy: $6.0 \mathrm{eV}$ for the krypton plasma and $8.0 \mathrm{eV}$ for the argon plasma, have a significant meaning for selection of the analytical line in GD-OES. As similar to the case of the nickel atomic lines (see Fig. 2), the intensities of cobalt atomic lines emitted from the krypton plasma less differ from those from the argon plasma. One can observe many Co I lines in the wavelength range of $330-390 \mathrm{~nm}$. They are assigned to the $3 d^{8} 4 p-3 d^{8} 4 s$ transition, whose excitation energies are 3.5-4.2 eV. Figure 5 shows calibration curves for two sensitive Co I lines, Co I $345.351 \mathrm{~nm}(4.02 \mathrm{eV})$ and
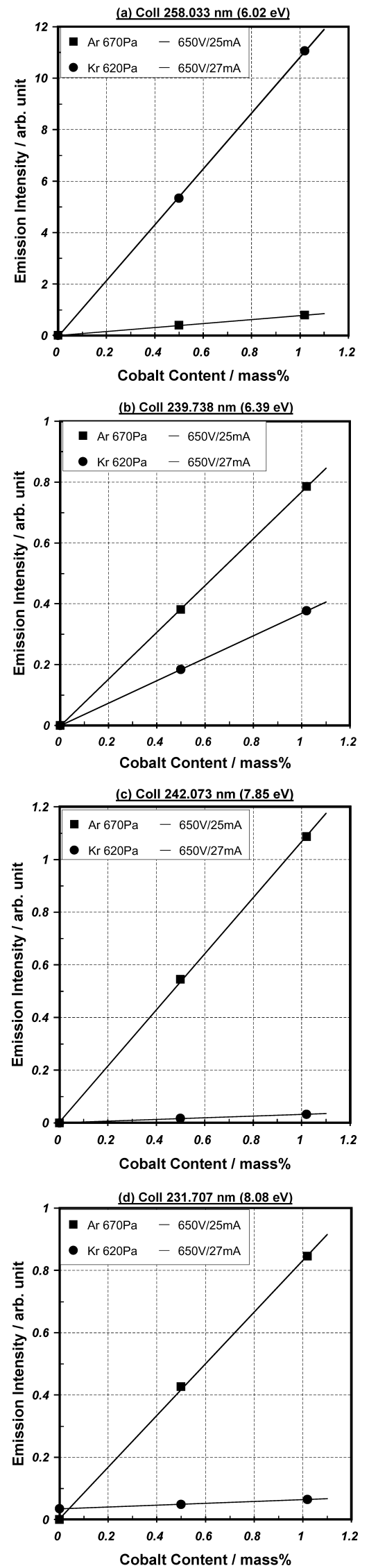

Fig. 4. Calibration curve for the Co II $258.033 \mathrm{~nm}$ (a), the Co II $239.738 \mathrm{~nm}$ (b), the Co II $242.073 \mathrm{~nm}$ (C), and the Co II $231.707 \mathrm{~nm}$ (d) in a krypton plasma (circle) and an argon plasma (square). Discharge conditions: $\mathrm{Kr}, 620 \mathrm{~Pa} / 650 \mathrm{~V} /$ $27 \mathrm{~mA}$; Ar, $670 \mathrm{~Pa} / 650 \mathrm{~V} / 25 \mathrm{~mA}$. 

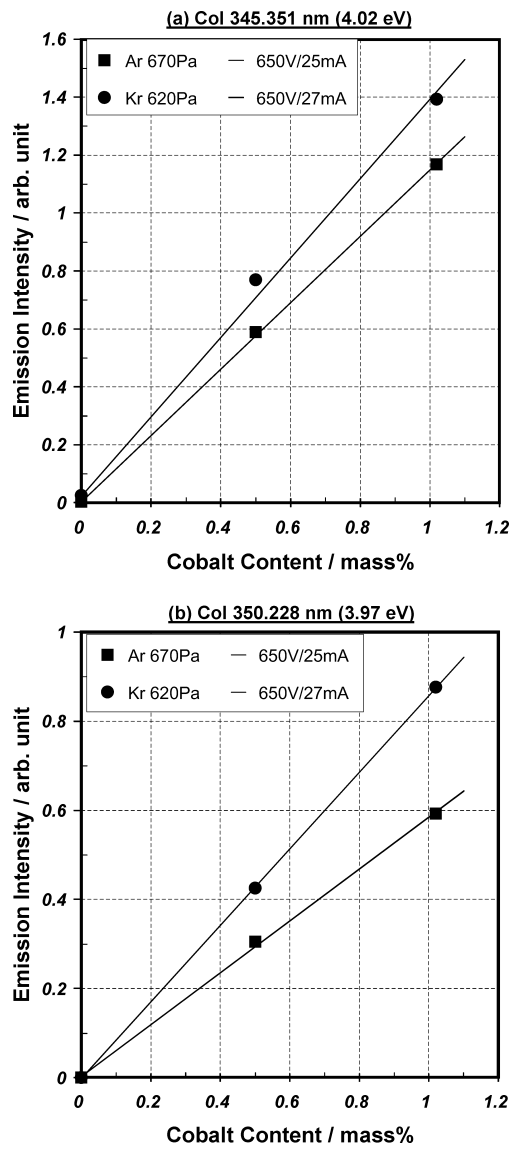

Fig. 5. Calibration curve for the CoI $345.351 \mathrm{~nm}$ (a) and the CoI $350.228 \mathrm{~nm}$ in a krypton plasma (circle) and an argon plasma (square). Discharge conditions: $\mathrm{Kr}, 620 \mathrm{~Pa} / 650 \mathrm{~V} /$ $27 \mathrm{~mA} ; \mathrm{Ar}, 670 \mathrm{~Pa} / 650 \mathrm{~V} / 25 \mathrm{~mA}$.

Co I $350.228 \mathrm{~nm}(3.97 \mathrm{eV})$, indicating that the sensitivity difference between these plasmas is much smaller than that of the Co II lines.

Vanadium has smaller first ionization potential than both nickel and cobalt. ${ }^{21)}$ Table 3 summarizes sensitive V II lines observed in the wavelength range of $239-320 \mathrm{~nm}$, when a pure vanadium sample is measured by using a krypton or an argon plasma at a gas pressure of $670 \mathrm{~Pa}$. They are assigned to the $3 d^{3} 4 p-3 d^{3} 4 s\left(3 d^{4}\right)$ transition or the $3 d^{3} 4 d-$ $3 d^{3} 4 p$ transition. $^{21)}$ The excitation energies of the former transition range from 4.29 to $7.70 \mathrm{eV}$ and those of the latter transition from 8.98 to $9.51 \mathrm{eV}$. Differing from the results of $\mathrm{Ni}$ II and Co II lines, few emission lines which are enhanced in the krypton plasma are found in Table 3. For instance, the emission intensity of the V II $266.321 \mathrm{~nm}(9.05 \mathrm{eV})$ was 4000 in the krypton plasma and 93000 in the argon plasma, and the intensity of the V II $309.310 \mathrm{~nm}(4.40 \mathrm{eV})$ was 13000 in the krypton plasma and 98000 in the argon plasma. Figure 6 shows variations in the emission intensities of these V II lines with increasing the discharge voltage, which clearly indicates that their emission intensities become much greater in the argon plasma than in the krypton plasma, independent of the applied discharge voltage. These results imply that there are no excited energy levels of vanadium ion which are suitable for the resonance energy transfer from krypton ion due to mismatch in their excitation energies. Figure 7 shows calibration curves for these VII lines, which clearly represents that the argon
Table 3. Observed vanadium ionic lines and their assignment together with the relative intensity in the Ar and the Kr plasmas

\begin{tabular}{|c|c|c|c|c|c|c|}
\hline \multirow{2}{*}{$\begin{array}{c}\text { Wavelength } \\
\qquad(\mathrm{nm})\end{array}$} & \multicolumn{4}{|c|}{ Assignment } & \multicolumn{2}{|c|}{ Relative Intensity $^{*}$} \\
\hline & \multicolumn{2}{|c|}{ Upper level (eV) } & \multicolumn{2}{|c|}{ Lower Level (eV) } & \multirow{2}{*}{$\begin{array}{c}\mathbf{K r} \\
10000\end{array}$} & \multirow{2}{*}{$\begin{array}{c}\text { Ar } \\
19000\end{array}$} \\
\hline V I 318.530 & (atomic line & or the stand & ard of normaliza & ion) & & \\
\hline V II 238.970 & $3 d^{3} 4 p^{3} \mathbf{D}_{1}$ & 6.2578 & $3 d^{3} 4 s^{3} \mathbf{F}_{2}$ & 1.0712 & 300 & 200 \\
\hline V II 247.952 & $3 d^{3} 4 p^{3} \mathbf{D}_{3}$ & 6.6860 & $3 d^{t^{3}} \mathbf{F}_{4}$ & 1.6873 & 6000 & 5000 \\
\hline V II 248.307 & $3 d^{3} 4 p^{3} \mathbf{D}_{1}$ & 6.6642 & $3 d^{4}{ }^{3} \mathbf{F}_{2}$ & 1.6726 & 5000 & 13000 \\
\hline V II 252.792 & $3 d^{3} 4 p^{3} \mathbf{H}_{6}$ & 6.4784 & $3 d^{4}{ }^{3} \mathbf{H}_{6}$ & 1.5753 & 700 & 1000 \\
\hline V II 252.884 & $3 d^{3} 4 p^{3} \mathbf{H}_{5}$ & 6.4661 & $3 d^{4} \mathbf{H}_{5}$ & 1.5648 & 6000 & 10000 \\
\hline V II 258.496 & $3 d^{3} 4 p^{3} \mathbf{G}_{5}$ & 6.8446 & $3 d^{3} 4 s^{3} \mathbf{G}_{5}$ & 2.0498 & 500 & 2000 \\
\hline V II 263.067 & $3 d^{3} 4 p^{3} \mathbf{F}_{4}$ & 6.5286 & $3 d^{+3} \mathbf{G}_{5}$ & 1.8170 & 8000 & 4000 \\
\hline V II 264.082 & $3 d^{3} 4 p^{5} \mathbf{H}_{3}$ & 8.9823 & $3 d^{3} 4 p^{5} \mathbf{G}_{2}$ & 4.2888 & 4000 & 46000 \\
\hline V II 264.433 & $3 d^{3} 4 p^{5} \mathbf{H}_{4}$ & 8.9950 & $3 d^{3} 4 p^{5} \mathbf{G}_{3}$ & 4.3078 & 3000 & 54000 \\
\hline V II 264.584 & $3 d^{3} 4 p^{3} \mathbf{F}_{2}$ & 6.4775 & $3 d^{+3} \mathbf{G}_{3}$ & 1.7930 & 2000 & 1000 \\
\hline V II 264.933 & $3 d^{3} 4 d^{5} \mathbf{H}_{5}$ & 9.0111 & $3 d^{3} 4 p^{5} \mathbf{G}_{4}$ & 4.3327 & 56000 & 70000 \\
\hline V II 265.565 & $3 d^{3} 4 d^{5} \mathbf{H}_{6}$ & 9.0305 & $3 d^{3} 4 p^{5} \mathbf{G}_{5}$ & 4.3633 & 4000 & 83000 \\
\hline V II 266.321 & $3 d^{3} 4 d^{5} \mathbf{H}_{7}$ & 9.0532 & $3 d^{3} 4 p^{5} \mathbf{G}_{6}$ & 4.3993 & 4000 & 93000 \\
\hline V II 275.955 & $3 d^{3} 4 d^{5} \mathbf{F}_{3}$ & 9.0688 & $3 d^{3} 4 p^{5} \mathbf{F}_{3}$ & 4.5773 & 2000 & 28000 \\
\hline V II 276.070 & $3 d^{3} 4 p^{1} \mathbf{I}_{6}$ & 6.8690 & $3 d^{+1} \mathbf{I}_{6}$ & 2.3794 & 2000 & 4000 \\
\hline V II 276.587 & $3 d^{3} 4 d^{5} \mathbf{G}_{4}$ & 9.0585 & $3 d^{3} 4 p^{5} \mathbf{F}_{3}$ & 4.5773 & 1000 & 1000 \\
\hline V II 277.137 & $3 d^{3} 4 d^{5} \mathbf{F}_{2}$ & 9.0540 & $3 d^{3} 4 p^{5} \mathbf{F}_{1}$ & 4.5817 & 2000 & 39000 \\
\hline V II 277.427 & $3 d^{3} 4 p^{3} \mathbf{G}_{3}$ & 6.0230 & $3 d^{4}{ }^{3} \mathbf{H}_{4}$ & 1.5554 & 300 & 1000 \\
\hline V II 277.775 & $3 d^{3} 4 p^{5} \mathbf{S}_{2}$ & 6.1658 & $3 d^{3} 4 s^{5} \mathbf{P}_{3}$ & 1.7037 & 1000 & 3000 \\
\hline V II 283.653 & $3 d^{3} 4 p^{3} \mathbf{G}_{5}$ & 6.0569 & $3 d^{t^{3}} \mathbf{F}_{4}$ & 1.6873 & 1000 & 2000 \\
\hline V II 284.758 & $3 d^{3} 4 p^{3} \mathbf{G}_{3}$ & 6.8623 & $3 d^{3} 4 s^{3} \mathbf{H}_{4}$ & 2.5097 & 25000 & 1000 \\
\hline V II 289.264 & $3 d^{3} 4 p^{5} \mathbf{D}_{2}$ & 4.6331 & $3 d^{3} 4 s^{5} \mathbf{F}_{3}$ & 0.3482 & 4000 & 13000 \\
\hline V II 289.332 & $3 d^{3} 4 p^{5} \mathbf{D}_{3}$ & 4.6519 & $3 d^{3} 4 s^{5} \mathbf{F}_{4}$ & 0.3680 & 3000 & 24000 \\
\hline V II 290.882 & $3 d^{3} 4 p^{5} \mathbf{D}_{4}$ & 4.6532 & $3 d^{3} 4 s^{5} \mathbf{F}_{5}$ & 0.3921 & 8000 & 44000 \\
\hline V II 292.401 & $3 d^{3} 4 p^{5} \mathbf{F}_{5}$ & 4.6310 & $3 d^{3} 4 s^{5} \mathbf{F}_{5}$ & 0.3921 & 9000 & 71000 \\
\hline V II 292.463 & $3 d^{3} 4 p^{5} \mathbf{F}_{4}$ & 4.6060 & $3 d^{3} 4 s^{5} \mathbf{F}_{4}$ & 0.3680 & 5000 & 64000 \\
\hline V II 309.310 & $3 d^{3} 4 p^{5} \mathbf{G}_{6}$ & 4.3993 & $3 d^{3} 4 s^{5} \mathbf{F}_{5}$ & 0.3921 & 13000 & 98000 \\
\hline V II 310.229 & $3 d^{3} 4 p^{5} \mathbf{G}_{5}$ & 4.3633 & $3 d^{3} 4 s^{5} \mathbf{F}_{4}$ & 0.3680 & 10000 & 79000 \\
\hline V II 311.069 & $3 d^{3} 4 p^{5} \mathbf{G}_{4}$ & 4.3327 & $3 d^{3} 4 s^{5} \mathbf{F}_{3}$ & 0.3432 & 8000 & 61000 \\
\hline V II 311.838 & $3 d^{3} 4 p^{5} \mathbf{G}_{3}$ & 4.3078 & $3 d^{3} 4 s^{5} \mathbf{F}_{2}$ & 0.3331 & 6000 & 44000 \\
\hline V II 312.527 & $3 d^{3} 4 p^{5} \mathbf{G}_{2}$ & 4.2888 & $3 d^{3} 4 s^{5} \mathbf{F}_{1}$ & 0.3229 & 4000 & 32000 \\
\hline V II 312.620 & $3 d^{3} 4 p^{5} \mathbf{G}_{4}$ & 4.3327 & $3 d^{3} 4 s^{5} \mathbf{F}_{4}$ & 0.3680 & 7000 & 20000 \\
\hline V II 313.026 & $3 d^{3} 4 p^{5} \mathbf{G}_{3}$ & 4.3078 & $3 d^{3} 4 s^{5} \mathbf{F}_{3}$ & 0.3482 & 2000 & 14000 \\
\hline V II 319.069 & $3 d^{3} 4 p^{3} \mathbf{F}_{4}$ & 5.0126 & $3 d^{3} 4 s^{3} \mathbf{F}_{4}$ & 1.1280 & 3000 & 12000 \\
\hline
\end{tabular}

*) Discharge conditions: Kr 530 Pa / 600 V / 36 mA; Ar 530 Pa / 600 V/ 33 mA.

plasma is generally superior to the krypton plasma for the vanadium determination. As shown in Fig. 7, there is no spectral interference from iron lines and gas emission lines. Although atomic lines of vanadium are observed in the wavelength range of $290-440 \mathrm{~nm}$, their intensities are generally weaker than those of the intense V II lines ${ }^{14)}$; therefore, they cannot be recommended as analytical lines in GD-OES.

The limit of determination (LOD) for the emission lines of nickel, cobalt, and vanadium, can be estimated from the corresponding calibration curves in the case of iron-matrix alloy samples. It was estimated from ten-times the standard deviation of their intensity fluctuations when using a pure iron sample. For the nickel determination in iron-based alloy samples, the most sensitive line is obtained when 

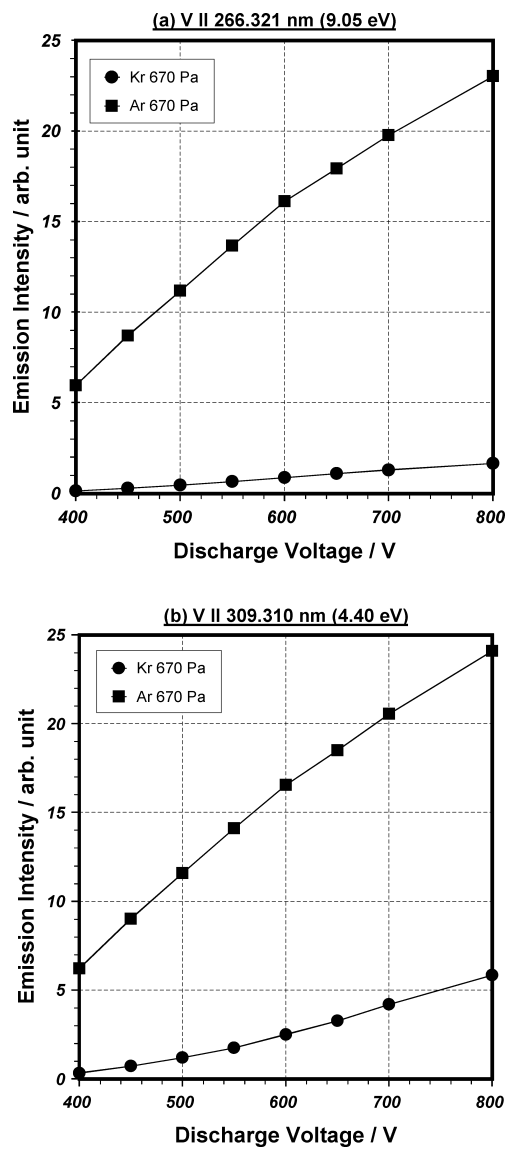

Fig. 6. Variations in the emission intensity of the V II $266.321 \mathrm{~nm}$ (a) and the VII $309.310 \mathrm{~nm}$ in a krypton plasma (circle) and an argon plasma (square). Sample: pure vanadium; gas pressure: $\mathrm{Kr} 670 \mathrm{~Pa}$ and $\mathrm{Ar} 670 \mathrm{~Pa}$.

krypton is employed as the plasma gas: the Ni II 230.299 $\mathrm{nm}$ has the best LOD of 0.013 mass\%. The Ni II 229.965$\mathrm{nm}$ line should be selected as the analytical line in the argon plasma; however, its LOD (0.053 mass\%) is inferior to that of the Ni II 230.299-nm line excited by the krypton plasma. The Ni I 352.453-nm line is the most sensitive of the Ni I lines in both the krypton and the argon plasmas. The LODs are estimated to be 0.012 mass $\%$ for the krypton plasma and 0.018 mass $\%$ for the argon plasma, which are as much as the LOD of Ni II 229.965-nm line excited by the krypton plasma. In the case of the iron-cobalt samples, the Co II $258.033 \mathrm{~nm}$ gives the best LOD of $0.007 \mathrm{mass} \%$ also when krypton is employed, whereas a different emission line whose LOD is 0.050 mass $\%$, the Co II $242.073-\mathrm{nm}$ line, is the most sensitive in the argon plasma. The CoI 345.351-nm line is the most sensitive of the CoI lines in both the krypton and the argon plasmas. However, the LOD $(0.040 \mathrm{mass} \%)$ is not beyond the LODs of several Co II lines excited by the krypton plasma. It is thus concluded that the krypton plasma is more recommended for the determination of nickel and cobalt if the most intense ionic lines are selected. On the other hand, the argon plasma is suitable for the determination of vanadium, because the V II $309.310 \mathrm{~nm}$ yields the best LOD of $0.008 \mathrm{mass} \%$ when argon is employed, whereas no sensitive V II lines appear in the krypton plasma. These results indicate that an appropriate selection of the plasma gas is the most important for improving the detection sensitivity in GD-OES.
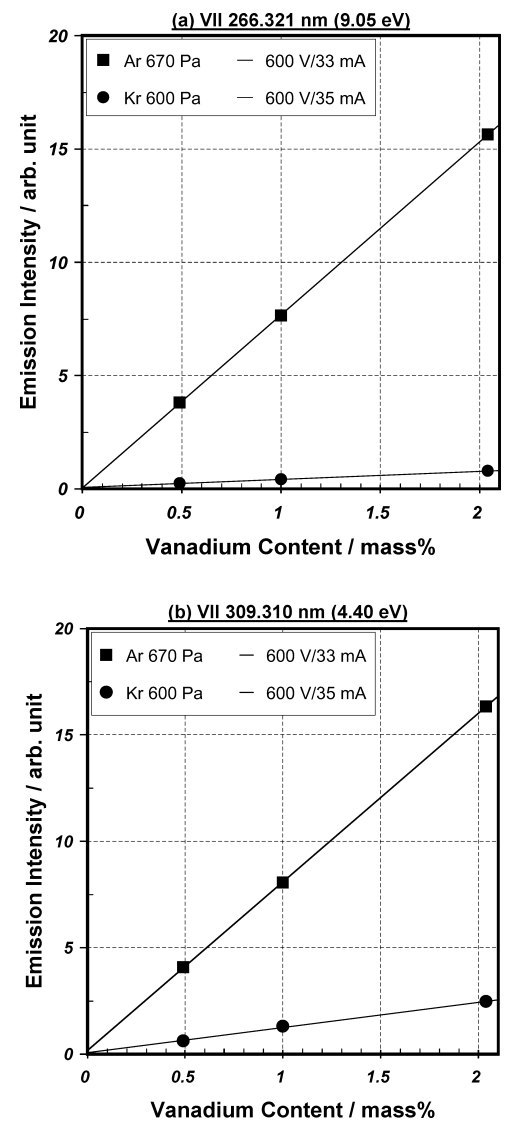

Fig. 7. Calibration curve for the V II $266.321 \mathrm{~nm}$ (a) and the V II $309.310 \mathrm{~nm}$ in a krypton plasma (circle) and an argon plasma (square). Discharge conditions: $\mathrm{Kr}, 600 \mathrm{~Pa} /$ $600 \mathrm{~V} / 35 \mathrm{~mA}$; Ar, $670 \mathrm{~Pa} / 650 \mathrm{~V} / 33 \mathrm{~mA}$.

\section{Conclusions}

The relative intensities of ionic lines of nickel, cobalt, and vanadium are largely dependent on the kind of the plasma gas in GD-OES. Different ionic lines of nickel and cobalt are predominantly excited between in the krypton plasma and in the argon plasma, whereas ionic lines of vanadium have larger emission intensities in the argon plasma rather than in the krypton plasma. In the determination of nickel and cobalt, the krypton plasma is recommended as the excitation source compared to the argon plasma, while the argon plasma is superior to the krypton plasma in the determination of vanadium.

\section{REFERENCES}

1) R. Payling, D. Jones, A. Bengtson (Eds.): Glow Discharge Optical Emission Spectrometry, John Wiley and Sons, Chichester, (1997).

2) R. K. Marcus and J. A. C. Broekaert (Eds.): Glow Discharge Plasmas in Analytical Spectroscopy, John Wiley and Sons, Chichester, (2003).

3) K. Wagatsuma and K. Hirokawa: Anal. Chem., 57 (1985), 2901.

4) M. Dogan, K. Laqua and H. Massmann: Spectrochim. Acta, 26B (1971), 631.

5) M. Dogan, K. Laqua and H. Massmann: Spectochim. Acta, 27B (1972), 65.

6) P. W. J. M. Boumans: Anal. Chem., 44 (1972), 1219.

7) R. Payling, D. G. Jones and S. A. Gower: Surf. Interface Anal., 20 (1993), 959.

8) W. Fischer, A. Nomads and H. Nickel: J. Anal. Atom. Spectrom., 9 (1994), 375. 
9) K. Wagatsuma and K. Hirokawa: Spectrochim. Acta, 42B (1987), 523.

10) K. Wagatsuma and K. Hirokawa: Anal. Chem., 60 (1988), 702.

11) K. Wagatsuma and K. Hirokawa: Spectrochim. Acta, 46B (1991), 269.

12) S. J. Christopher, M. L. Hartenstein, R. K. Marcus, M. Belkin and J. A. Caruso: Spectrochim. Acta, 53B (1998), 1181.

13) M. Belkin, J. A. Caruso, S. J. Christopher and R. K. Marcus: Spectrochim. Acta, 53B (1998), 1197.

14) K. Wagatsuma: Bunseki Kagaku, 48 (1999), 457.

15) K. Wagatsuma and H. Honda: Spectrochim. Acta, 60B (2005), 1538.
16) E. B. M. Steers and R. J. Fielding: J. Anal. Atom. Spectrom., 2 (1987), 239.

17) O. S. Duffendach and J. G. Black: Phys. Rev., 34 (1929), 35.

18) K. Wagatsuma and K. Hirokawa: Surf. Interface Anal., 6 (1984), 34.

19) H. Morita, K. Wagatsuma and K. Hirokawa: Surf. Interface Anal., 17 (1991), 116.

20) W. Grimm: Spectrochim. Acta, 23B (1968), 443

21) J. Sugar and C. Corliss: J. Phys. Chem. Ref. Data, 14 (1985), Suppl. No. 2.

22) K. Wagatsuma: Bioanal. Anal. Chem., 393 (2009), 2067. 